\title{
Asymmetric energy transfers in driven nonequilibrium systems and arrow of time
}

\author{
Mahendra K. Verma ${ }^{1, *}$ \\ ${ }^{1}$ Department of Physics, Indian Institute of Technology, Kanpur, India 208016
}

\begin{abstract}
Fundamental interactions are either fully or nearly symmetric under time reversal. But macroscopic phenomena have a definite arrow of time. Though there is no convergence on the origin of time's preferential direction, many researchers believe that the direction of time is towards increasing entropy. In this paper, we provide an alternate point of view. In driven-dissipative nonequilibrium systems forced at large scale, the energy flows from large scales to dissipative scales. This generic and multiscale process breaks time reversal symmetry and principle of detailed balance, thus can yield an arrow of time. In this paper we propose that conversion of large-scale coherence to smallscales incoherence could be treated as a dissipation mechanism for generic physical systems. We illustrate the above processes using turbulence as an example. We argue that the above picture of time irreversibility could be employed to the universe and to many-body quantum systems.
\end{abstract}

\section{INTRODUCTION}

Fundametal forces-gravity, electrodynamics, and strong nuclear - exhibit time reversal symmetry. Weak nuclear force however exhibits a small violation of this symmetry. Hence, from the perspectives of fundamental interactions, forward and backward motion of a physical system are indistinguishable apart from a small violation for weak nuclear force [1-3]. This observation contradicts daily experience where physical and biological systems evolve forward in time. For example, stars, planets, and living beings take birth, live, and then die. There have been many attempts to explain this asymmetry, some of which will be summarized below. In this paper, we show how asymmetric energy transfers determine the arrow of time in driven-dissipative nonequilibrium systems. We illustrate the properties of energy transfers for hydrodynamic turbulence, which is a well-studied example of driven-dissipative nonequilibrium system.

There is a general consensus that the arrow of time in physical systems arises due to the second law of thermodynamics, according to which a nonequilibrium system evolves in such a way that the entropy of the system always increases [1-3]. For example, when we mix a set of cold molecules with another set of hot molecules, the mixture tends towards a uniform distribution of molecules with maximum entropy. The other mechanisms invoked for explaining arrow of time are sensitivity to initial condition in nonlinear systems [4], measurements in quantum systems [5], chaos induced decoherence [6], etc. The energy transfer mechanism presented in this paper is an alternative framework to break the time reversal symmetry.

Some of the leading examples of driven-dissipative nonequilibrium systems are turbulence, earthquakes, crack propagation, fragmentation, free market economy, astrophysical flows, etc. Several common features among them are (a) energy supply at large scales; (b) energy cascade from large scale to intermediate scale and then to

\footnotetext{
*mkv@iitk.ac.in
}

small scale; (c) dissipation at small scales; (d) multiscale physics with energy transfers across scales [7-9]. Each of the above systems are covered in vast literature. Here, we take turbulence as an illustrative example because it has been widely studied, and it is familiar to physicists.

Unidirectional energy transfers (from large scales to small scales) break the time reversal symmetry in turbulence. In a time-reversed version of a turbulent flow, the energy will flow from small scales to large scales, contrary to real systems. Jucha et al. [10] made the above observation, and then went on to quantify the irreversibility in turbulence using the relative motion between two particles. They showed that the difference between the forward and backward dispersion of the two particles varies as $t^{3}$, where $t$ is the time elapsed. Davidson [11] attributed the arrow of time in a turbulent flow to chaotic advection arising due to the nonlinear terms.

In the present paper we focus on the energy transfers in turbulence and show how they play a critical role in determining the arrow of time. In turbulence, the energy transfers break the principle of detailed balance due to asymmetric energy transfers from large scales to small scales. We show that the above scenario is applicable to many nonequilibrium systems. Also, we argue that the dissipation of kinetic energy at small scales and its subsequent conversion to heat offers an interesting recipe for introducing dissipation in a generic multiscale system. In the paper we also emphasise that the above multiscale description involving energy transfers differs significantly from the prescription of second law of thermodynamics. Towards the end of the paper we argue how the ideas of energy transfers could be extended to the universe and many-body quantum systems.

The outline of the paper is as follows: In Sec. II, we describe how asymmetric energy transfers in turbulence can set the direction of time. In Sec. III we argue that the conversion of large-scale coherent energy to small-scale incoherent energy could be treated as a generic dissipation mechanism in nonequilibrium systems. We conclude in Sec. IV. 


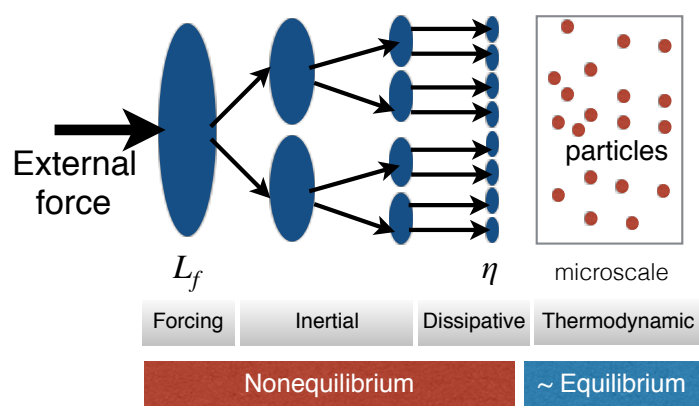

(a)

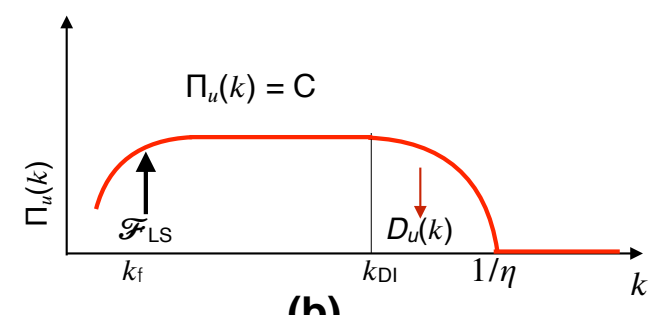

(b)

FIG. 1. (color online) Schematic diagrams illustrating energy transfers in three-dimensional hydrodynamic turbulence, which is an example of a driven-dissipative nonequilibrium system: (a) The energy supplied at large scales cascades to the inertial range and then to the dissipative range. The dissipation of coherent kinetic energy at the dissipative scales heats up the particles at microscale. The energy transfers are indicated by arrows. The structures at the forcing and inertial range are governed by nonequilibrium processes, while those at microscale are in approximate equilibrium. (b) Plot of spectral energy flux $\Pi_{u}(k)$ vs. $k$. $\Pi_{u}(k) \sim$ const in the inertial range, and it decreases with $k$ in the dissipative range. $\mathcal{F}_{\mathrm{LS}}$ is the energy supply by the external force, and $D_{u}(k)$ is the dissipation rate. There is no energy flux at the microscales due to the detailed balance.

\section{DIRECTION OF TIME IN TURBULENT SYSTEMS}

In this section we describe the essential physics of turbulence, and then the arrow of time in such flows. Consider an incompressible hydrodynamic flow that is forced at large length scales $\left(L_{f}\right.$, forcing scale). According to the celebrated Kolmogorov's theory [7, 8], in a turbulent flow, the energy supplied at large scales cascades to intermediate scales (called inertial range) and then to dissipative scales. See Fig. 1a for an illustration. Note that the energy supply rate, the energy flux in the inertial range, and the energy dissipation rate are all equal (denoted by $\epsilon_{u}$ ) $[7,9,12,13]$.

In the above macroscopic picture, which is based on continuum approximation, the velocity field at a position $\mathbf{r}$ is averaged over many microscopic particles around $\mathbf{r}$. This description of the velocity field is sensible up to Kolmogorov length scale $\eta$, which is approximately equal to $\left(\nu / \epsilon_{u}^{3}\right)^{1 / 4}$, where $\nu$ is the kinematic viscosity of the fluid.
The microscopic physics, e.g. kinetic theory, describes the dynamics of particles at length scales smaller than $\eta$ because the particles at these scales are under an approximate equilibrium. However, the system is dynamic and in a nonequlibrium state for $l>\eta$ (see Fig. 1a). Thus, the length scale $\eta$ plays a major role in separating the coherent hydrodynamic structures with random motion at microscopic scales. We illustrate these features using an example. When we pour milk in a coffee cup, the large fluid structures of milk successively breaks into a hierarchy of structures, until it diffuses at microscales where the macroscopic coherent energy is destroyed as disorder or heat.

It is customary to describe turbulence in Fourier space too with wavenumber $k \sim 1 / l$. In this description, as shown in Fig. 1b, the energy flux (or energy cascade rate) $\Pi_{u}(k) \sim$ const in the inertial range, and it dampens in the dissipative range $k_{\mathrm{DI}}<k<1 / \eta$. There is no energy flux in the microscopic range.

The nonlinear term of the Navier-Stokes equation, $\mathbf{u} \cdot \nabla \mathbf{u}$ where $\mathbf{u}$ is the velocity field, induces energy transfers from one scale to another scale. In Fourier space, the basic unit of nonlinear interactions is a wavenumber triad (k, p, q) satisfying $\mathbf{k}=\mathbf{p}+\mathbf{q}$. Dar et al. [14] and Verma [15] showed that the energy transfer from Fourier mode $\mathbf{u}(\mathbf{p})$ to Fourier mode $\mathbf{u}(\mathbf{k})$ under the mediations of Fourier mode $\mathbf{u}(\mathbf{q})$ is given by

$$
S^{u u}(\mathbf{k}|\mathbf{p}| \mathbf{q})=\Im\left[\{\mathbf{k} \cdot \mathbf{u}(\mathbf{q})\}\left\{\mathbf{u}(\mathbf{p}) \cdot \mathbf{u}^{*}(\mathbf{k})\right\}\right] .
$$

Using the above function we define the energy flux $\Pi_{u}\left(k_{0}\right)$, which is the energy emanating from a wavenumber sphere of radius $k_{0}$ due to nonlinear interactions, as:

$$
\Pi_{u}\left(k_{0}\right)=\sum_{|\mathbf{p}| \leq k_{0}} \sum_{|\mathbf{k}|>k_{0}} S^{u u}(\mathbf{k}|\mathbf{p}| \mathbf{q}) .
$$

A nonzero flux implies that the phases of the Fourier modes are coherently organised. The flux would be zero if the phases of the Fourier modes are random and uncorrelated. Kolmogorov [7, 8] and Pao [16] showed that the energy spectrum of the inertial-dissipation range is given by

$$
\begin{aligned}
& E_{u}(k) \approx K_{\mathrm{Ko}} \epsilon_{u}^{2 / 3} k^{-5 / 3} \exp \left(-\frac{3}{2} K_{\mathrm{Ko}}(k \eta)^{4 / 3}\right), \\
& \Pi_{u}(k) \approx \epsilon_{u} \exp \left(-\frac{3}{2} K_{\mathrm{Ko}}(k \eta)^{4 / 3}\right),
\end{aligned}
$$

where $k=|\mathbf{k}|$, and $K_{\mathrm{Ko}}$ is Kolmogorov's constant whose value is approximately 1.6 [9]. The aforementioned Kolmogorov's spectrum and flux have been verified by many experiments and numerical simulations. For a recent numerical work, refer to Verma et al. [17].

Now we investigate in some detail the energy transfers and flux in a three-dimensional turbulent flow. It has been shown that for a turbulent flow [15, 18],

$$
\begin{gathered}
S^{u u}(\mathbf{k}|\mathbf{p}| \mathbf{q})>0 \text { for } k>p, \\
S^{u u}(\mathbf{k}|\mathbf{p}| \mathbf{q})<0 \text { for } k<p .
\end{gathered}
$$


Hence, energy flows preferentially from large scales to small scales, thus breaking the time reversal symmetry as well as the detailed balance (of energy transfer). The energy flux, which is a sum of $S^{u u}$ 's [Eq. (4)], is positive in the inertial range. These energy transfers create and sustain a hierarchy of structures. Note that in a time-reversed system, $\mathbf{u} \rightarrow-\mathbf{u}$, substitution of which in Eqs. $(1,2)$ leads to opposite $S^{u u}(\mathbf{k}|\mathbf{p}| \mathbf{q})$ and negative $\Pi_{u}(k)$, implying flow of energy from small scales to large scales. Clearly, such a process is not allowed from physical considerations (Kolmogorov's theory of turbulence), hence the time-reversed flow can be differentiated from the real flow. Thus, the energy transfers and flux set the arrow of time in a turbulent system.

For a steady incompressible turbulent flow, the energy contents of structures at the large and intermediate scales remain unchanged on an average. Hence the measure of disorder (entropy) is not expected to increase for these structures. Thus, entropy is not very important for the description of steady-state turbulent structures of an incompressible flow. However, the energy supplied at large scales is finally transferred to the molecules at microscales. Hence the molecules at the microscopes get heated up, thus increasing the entropy at these scales. We also remark that the dynamics (Navier-Stokes equations) and viscous dissipation play a key role in turbulence and driven-dissipative nonequilibrium systems. Broadly speaking, the forcing and inertial range, as well as part of dissipation range, would be in nonequilibrium state; and a fraction of dissipation range and all of microscopic range would be in an approximate equilibrium state. See Fig. 1 for an illustration. We remark that turbulence does not violate second law of thermodynamics. Here, we argue that the unidirectional energy transfers in turbulence provide an alternative viewpoint for time reversal asymmetry.

The aforementioned transfers from large scales to small scales are observed in many driven-dissipative nonequilibriun systems - for examples in earthquakes [19], magnetohydrodynamic turbulence [15], scalar turbulence and thermal convection [20-22], finance [23], and astrophysical and geophysical flows [24, 25]. In thermal convection, large-scale thermal plumes drive the turbulence. Galactic and stellar turbulence have behaviour similar to hydrodynamic turbulence with energy feed at large scale by supernovas and star core (where nuclear reaction takes place) respectively [26]. Collisions of tectonic plates feed energy to earthquakes, and this energy is transmitted to smaller scales. The money supply at large scales drives a free market economy. The energy transfers in the above systems differ in detail, but energy flows from large scales to small scales in all of them. This crucial feature sets the arrow of time in these nonequilibrium systems.

Exceptions to the aforementioned picture are twodimensional and quasi-two-dimensional (e.g. rotating) turbulence where the energy flows from small scales to large scales $[11,13,27,28]$ due to an inverse cascade of energy. These transfers lead to formation of large scale structures. Such systems do not violate our model for the direction of time. If our system is two-dimensional and quasi-two-dimensional turbulence, then we define the forward direction of time as one in which the energy transfers are from small scales to large scale. We have to carefully observe both the space dimensionality and energy transfers for determining the direction of time.

We remark that the viscous dissipation at small scales plays an important role in setting the direction of energy flow in driven-dissipative nonequilibrium systems. Typically, the energy supplied at large scales flows towards the energy sink at the dissipative scale. Given this, an important question is how to introduce an effective dissipation in a nonequilibrium system. Here too, the physics of turbulence gives importance clues, as described in the next section.

\section{HOW TO INCORPORATE DISSIPATION IN A PHYSICAL SYSTEM?}

Fundamental forces of nature are conservative or nondissipative. Hence, inclusion of dissipation appears impossible from the perspectives of fundamental physics. Yet, multiscale description of turbulence provides interesting possibilities for an inclusion of dissipation in a physical system.

An important question: what is the origin of the viscous dissipation in a fluid flow when it is composed of many interacting molecules and atoms, as visualised in statistical mechanics? Naively, we do not expect any dissipation in a flow. The answer however becomes apparent when we think in terms of multiscale physics, and separate the coherent and incoherent structures. As shown in Fig. 1, in a turbulent flow, the relative velocity between the fluid structures causes successive cascade of coherent energy to smaller and smaller scales. Finally, the coherent structures are fully dissipated at Kolmogorov's microscale $(\eta)$. Hence, in hydrodynamic turbulence, viscous dissipation provides an interesting model for transferring energy from coherent structures to incoherent ones. We emphasize that in a decaying turbulence (with no external force), the total kinetic energy of the flow, $\rho u^{2} / 2$ where $\rho$ is the fluid density, decays. But the total energy, which is a sum of kinetic energy of the fluid and particles, is conserved. Thus, the viscosity converts coherent kinetic energy to thermal energy at the microscales. Similar processes occur in plasma turbulence where the energy supplied from the large-scale velocity and magnetic fields are dissipated at microscales by kinetic processes involving charge particles $[29,30]$.

We expect a similar separation of scales in many driven-dissipative nonequilibrium systemsastrophysical turbulence (in stars, galaxies, galaxy clusters, etc.), free market economy with cascade of money, earthquakes, etc. The energy (or similar quantity, e.g. money in a financial system) flows from large scales to small scales, where it is dissipated. The physical 
processes at small scales that converts coherent energy to incoherent ones could be treated as dissipation. Even the frictional loss during the motion of a block on a surface can be treated as conversion of coherent kinetic energy of the block to the incoherent heat energy of molecules at the interface. Introduction of dissipation in the universe and many-body (macroscopic) quantum systems appears to be a possibility under this perspective.

The universe is very complex due to intricate spacetime structures, complicated and enormous energy sources and sinks, unknown initial condition (during the early universe), etc. Gravitational interactions are conservative. Yet, from hydrodynamic perspectives, it is possible to treat the universe as a driven-dissipative nonequilibrium system with energy flowing from large scales (driven by sources like supernova) to intermediate scales and then to small scales. For example, largescale kinetic energy of a galaxy is transferred to the motion of dust and/or charged particles. Such mechanisms are employed for the generation of magnetic field in the universe [26]. Note however that dynamical interactions among the entities could lead to formation or destruction of structures. For example, depending on the forces, (local) space dimensionality, and initial configurations, the flow could become less structured or more structured. As described in Section II, large-scale structures are formed in two-dimensional or quasi-two-dimensional turbulence [11, 13, 27, 28]. Note that strong rotation [28], as in galaxies, or buoyancy due to density-stratification [22], as in planetary and stellar atmospheres, can make the flow quasi-two-dimensional. Hence, the aforementioned dynamical and multiscale perspective with energy transfers could possibly provide a cosmological arrow of time for the universe (even in the scenario of collapsing universe).

Dissipation or decoherence in quantum systems is quite intriguing, and it remains primarily an unsolved problem. Some of the proposed mechanisms for quantum decoherence are collapse of wave function during a measurement, interaction with enviornment [5], chaos [6], etc. In this short article, we do not delve into these topics, but describe recent observations on dissipation in quantum turbulence. Recent experiments and numerical simulations on superfluid turbulence allude to certain similarities between quantum turbulence and classical Navier-Stokes turbulence [31]. For a wavenumber band, $E_{u}(k) \sim k^{-5 / 3}$ with a constant energy cascade. Bradley et al. [32] argued that energy dissipation in Helium-3 superfluid turbulence, which is quantum many-body system, occurs via phonon coupling. Thus microscopic processes like phonon interactions in Helium-3 could provide dissipation of large-scale quantum correlations (also see [33]). It would be interesting to attempt similar ideas for other many-body quantum systems, such as Bose-Einstein condensate, quantum cavity with many atoms, etc.

Thus, a conversion of (cascaded) coherent energy to incoherent one at small scales provide an interesting way to incorporate dissipation in a driven-dissipative nonequilibrium system, as well as in many-body classical and quantum systems. This idea however needs further explorations using experiments and numerical simulations.

\section{CONCLUSIONS}

In summary, unidirectional and multiscale energy transfers in turbulence and in driven-dissipative nonequilibrium systems provide an alternate formulation for determining the arrow of time in such systems. In this framework, dissipation at small scales and energy supply at large scales play a critical role. An important outcome of such formalism is a recipe to introduce dissipation in multiscale systems. We propose that the transformation from coherent energy to incoherent energy could be treated as dissipation in physical systems.

Interestingly, many biological systems too exhibit multiscale processes with energy supply at large scales, sustenance at intermediate scale, and dissipation at small scales. For example, a living being receives food at large scales, which is used by organs (all the way to cells in a hierarchal manner) to generate energy and nutrition at intermediate scales. The waste products (e.g. $\mathrm{CO}_{2}$ ) generated at small scales are excreted out. These timeasymmetric processes may be playing a key role in determining biological arrow of time.

Before closing the discussion we remark that the mechanism for breaking time reversal symmetry based on unidirectional energy transfers is very different from those proposed in second law of thermodynamics, and in chaos theory. According to the second law, an increase in entropy sets the direction of time. Note that the energy transfer formalism described in this paper does not involve entropy computations of fluid structures. Some researchers attribute the arrow of time in chaotic systems to sensitivity to initial condition. In a chaotic system, most initial conditions take the system to chaotic configurations with higher entropy, and only a small set of initial conditions evolve to ordered states. This mechanism of chaotic dynamics differs significantly from that involving energy transfers in a driven-dissipative nonequilibrium system.

We hope that the aforementioned multiscale framework may be useful for resolving some of the longstanding issues on the arrow of time in physical and biological systems, as well as in cosmology.

\section{ACKNOWLEDGMENTS}

The author thanks Roshan Samuel, Anurag Gupta, Anand Jha, and Arul Lakshminarayan for useful discussions. 
[1] R. P. Feynman, The Character of Physical Law (Modern Library, New York, 1994).

[2] J. L. Lebowitz, Rev. Mod. Phys. 71, S346 (1999).

[3] S. Carroll, From Eternity to Here (Oneworld Publications, 2011).

[4] S. H. Strogatz, Nonlinear Dynamics and Chaos: With Applications to Physics, Biology, Chemistry, and Engineering (Perseus Books, Reading MA, 2014), 2nd ed.

[5] J. A. Wheeler and W. H. Zurek, Quantum Theory and Measurement (Princeton University Press, 2014).

[6] M. V. Berry, in Quantum Mechanics: scientific perspectives on divine action, edited by R. J. Russel, P. Clayton, K. Wegter-McNelly, and J. Polkinghorne (Vatican Observatory CTNS publications, 2001), p. 41.

[7] A. N. Kolmogorov, Dokl Acad Nauk SSSR 32, 16 (1941).

[8] A. N. Kolmogorov, Dokl Acad Nauk SSSR 30, 301 (1941).

[9] U. Frisch, Turbulence: The Legacy of A. N. Kolmogorov (Cambridge University Press, Cambridge, 1995).

[10] J. Jucha, H. Xu, A. Pumir, and E. Bodenschatz, Phys. Rev. Lett. 113, 054501 (2014).

[11] P. A. Davidson, Turbulence: An Introduction for Scientists and Engineers (Oxford University Press, Oxford, 2004).

[12] L. D. Landau and E. M. Lifshitz, Fluid Mechanics, Course of Theoretical Physics (Elsevier, Oxford, 1987), 2nd ed.

[13] M. Lesieur, Turbulence in Fluids (Springer-Verlag, Dordrecht, 2008).

[14] G. Dar, M. K. Verma, and V. Eswaran, Physica D 157, 207 (2001).

[15] M. K. Verma, Phys. Rep. 401, 229 (2004).

[16] Y.-H. Pao, Phys. Fluids 11, 1371 (1968).
[17] M. K. Verma, A. Kumar, P. Kumar, S. Barman, A. G. Chatterjee, R. Samtaney, and R. Stepanov, Fluid Dyn. 53, 728 (2018) [arXiv:1705.04917].

[18] M. K. Verma, A. Ayyer, O. Debliquy, S. Kumar, and A. V. Chandra, Pramana-J. Phys. 65, 297 (2005).

[19] D. L. Turcotte, Fractals and Chaos in Geology and Geophysics (Cambridge University Press, 1997).

[20] P. K. Yeung and K. R. Sreenivasan, J. Fluid Mech. 716, R14 (2013).

[21] B. I. Shraiman and E. D. Siggia, Nature 405, 639 (2000).

[22] M. K. Verma, Physics of Buoyant Flows: From Instabilities to Turbulence (World Scientific, Singapore, 2018).

[23] M. K. Verma, in New Perspectives and Challenges in Econophysics and Sociophysics, edited by F. Abergel, B. Chakrabarti, A. Chakraborti, N. Deo, and K. Sharma (Springer, 2019) [arXiv:cond-mat.statmech].

[24] M. L. Goldstein and D. A. Roberts, Annu. Rev. Astron. Astrophys. 33, 283 (1995).

[25] J. C. McWilliams, Fundamentals of geophysical fluid dynamics (Cambridge University Press, Cambridge, 2006).

[26] Y. B. Zeldovich, A. A. Ruzmaikin, and D. D. Sokoloff, Magnetic fields in astrophysics (Gordon and Breach, 1983).

[27] G. Boffetta and R. E. Ecke, Annu. Rev. Fluid Mech. 44, 427 (2012).

[28] M. K. Sharma, A. Kumar, M. K. Verma, and S. Chakraborty, Phys. Fluids 30, 045103 (2018).

[29] B. G. Elmegreen and J. Scalo, Annu. Rev. Astron. Astrophys. 42, 211 (2004).

[30] M. K. Verma, J. Geophys. Res.-Space 101, 27543 (1996).

[31] S. K. Nemirovskii, Phys. Rep. 524, 85 (2012).

[32] D. I. Bradley, D. O. Clubb, S. N. Fisher, A. M. Guénault, R. P. Haley, C. J. Matthews, G. R. Pickett, V. Tsepelin, and K. Zaki, Phys. Rev. Lett. 96, 035301 (2006).

[33] E. Fonda, K. R. Sreenivasan, and D. P. Lathrop, PNAS 116, 1924 (2019). 